\title{
Transmission Electron Microtomography (TEMT) of Nanostructured Polymers and Polymer Nanocomposites
}

\author{
R. J. Spontak, $*$ A. O. Gozen,* M. B. Braunfeld,** D. A. Agard** \\ * Departments of Chemical \& Biomolecular Engineering and Materials Science \& Engineering, \\ North Carolina State University, Raleigh, NC 27695 \\ ** Department of Biochemistry \& Biophysics and Howard Hughes Medical Institute, University of \\ California, San Francisco, CA 94143
}

Numerous emerging nanotechnologies exploit the morphological characteristics and/or properties of nanostructured polymers or polymers into which inorganic species are either chemically grown or physically dispersed. Microphase-ordered block copolymers, macromolecules capable of spontaneously self-organizing into a variety of periodic and nonperiodic morphologies possessing a characteristic length scale typically on the order of tens of nanometers, constitute excellent examples of nanostructured polymers under consideration for nanotechnological purposes [1]. Hybrid organic/inorganic materials composed of a polymer and nanoscale objects (particles, plates, rods and tubes) with at least one characteristic length scale measuring on the order of a few to tens of nanometers are commonly referred to as nanocomposites and have received considerable attention due to their unique, and sometimes unexpected, properties [2].

The spatial characteristics of nanostructured polymers and polymer nanocomposites are crucial in dictating the ultimate thermal, mechanical, optical and transport properties of such advanced materials and must be fully characterized in 3D to understand their role in property development and stability. While methods employing progressive (layer-by-layer) etching in conjunction with either surface probe microscopy [3] or field-emission scanning electron microscopy [4] have been introduced for this intention, TEMT has provided the most detailed 3D analysis of nanostructured polymers and polymer nanocomposites to date. The present work will provide a timely overview of TEMT in the investigation of various "soft" materials possessing nanoscale morphological features.

Our previous studies of block copolymer systems self-organizing into periodic [5] and nonperiodic [6] bicontinuous morphologies have demonstrated that the local and global topological characteristics of such morphologies can be fully quantitated from TEMT volume elements reconstructed (by the filtered back-projection technique) from digital image sets with sub-pixel alignment error, thereby yielding unprecedented direct measurement of, for instance, interfacial curvature/area, coordination and genus. As demonstrated earlier [6], the principal advantage of TEMT over other strategies developed to elucidate the 3D characteristics of nanostructured polymers and polymer nanocomposites is its ability to provide quantifiable information with regard to nonperiodic morphologies. The images shown in Fig. 1 have been acquired from a macro/microphase-separated block copolymer/homopolymer blend exhibiting both periodic and nonperiodic channels of the minor component in a matrix of the major component. Topological analysis of these data reveals that the coexisting morphologies only differ in their genus. A single section of the reconstruction of a templated block polymer is displayed with its $3 \mathrm{D}$ volume element in Fig. 2. Time-dependent nanostructured polymer morphologies, as well as discrete shapes (such as nanotubes), will also be presented and discussed in this overview. The serial sections provided in Fig. 3 are obtained from a polymer nanocomposite and indicate the spatial relationship between inorganic (colloidal silica, 
CS) nanoparticles capable of hydrogen-bonding and $\sim 10 \mathrm{~nm}$ nanofibrils generated by the selforganization of hydrogen-bonding gelator molecules added to a polymer matrix [7]. Other types of nanocomposites and the utility of TEMT in their 3D characterization will likewise be described.

\section{References}

[1] C. Park, J. Yoon and E. L. Thomas, Polymer 44 (2003) 6725.

[2] T. C. Merkel et al., Science 296 (2002) 519.

[3] C. Harrison et al., Polymer 39 (1998) 2733.

[4] R. Magerle, Phys. Rev. Lett. 85 (2000) 2749.

[5] H. Jinnai et al., Phys. Rev. Lett. 84 (2000) 518.

[6] H. Jinnai et al., Adv. Mater. 14 (2002) 1615.

[7] E. A. Wilder et al., J. Phys. Chem. B 107 (2003) 11633.

[8] This work was supported by the U.S. Department of Energy under Contract No. DE-FG0299ER14991, the National Science Foundation under Contract No. CHE-9876674, and the Research Council of Norway under the NANOMAT Program. We thank Dr. H. Jinnai (Kyoto Institute of Technology) for technical assistance and Dr. H. Hasegawa (Kyoto University) for the blend.

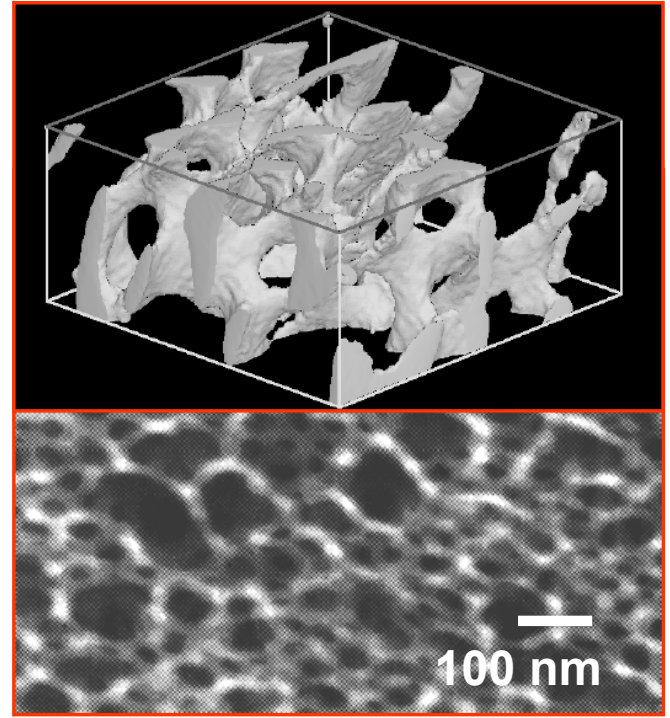

Fig. 1. Nonperiodic polymer morphology in a block copolymer/homopolymer blend.

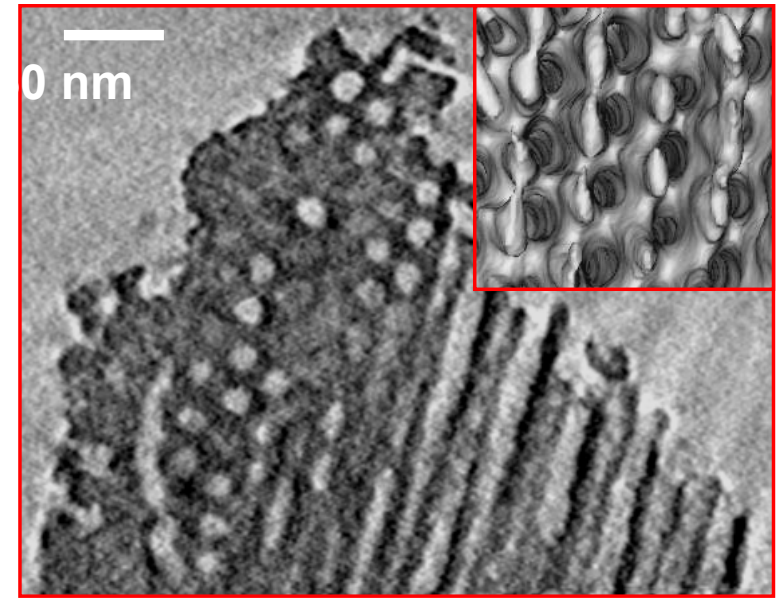

Fig. 2. Porous silica generated by templating a nanostructured block copolymer. The main spherical (hole) morphology is seen in the 3D volume (inset). Channels are also evident.

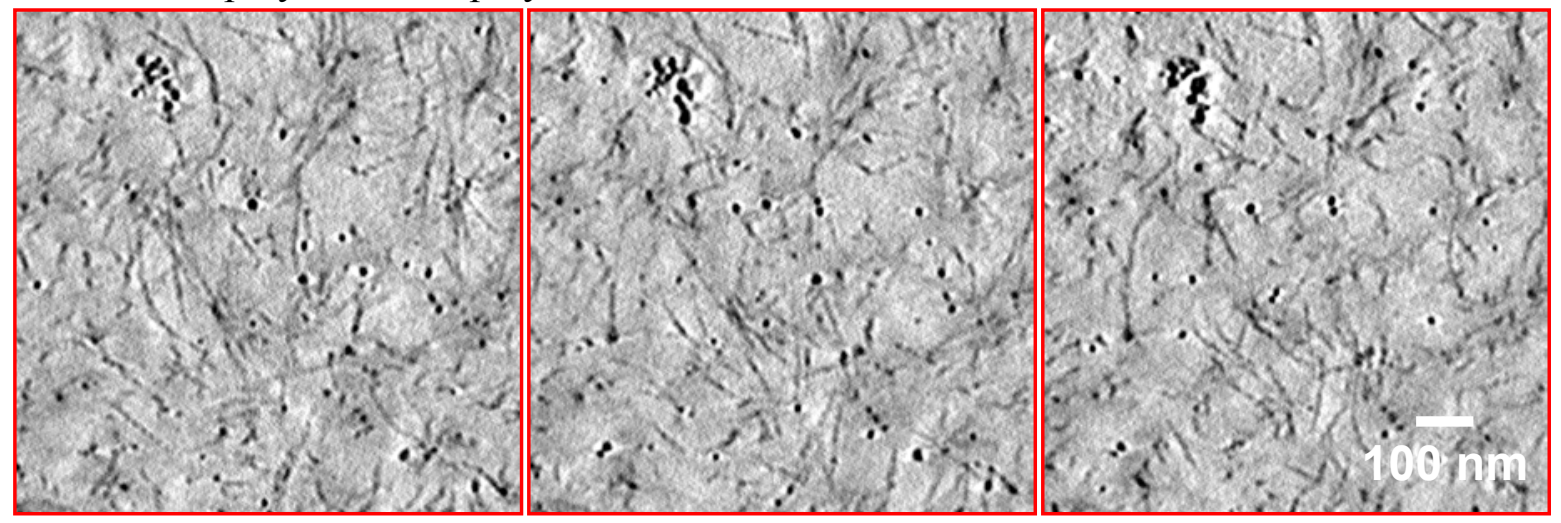

Fig. 3. Sequential sections of a TEMT reconstruction showing nanofibrils and CS nanoparticles. 